\title{
The Hammond Electric Organ
}

$\mathrm{T}$ HE development of the loud-speaker, and more especially the demonstration of its capability of reproducing all types of music in connexion with the projection of the talking film in the cinemas, has suggested to a number of inventors the possibility of the replacement of the ordinary pipe organ by one which is entirely electric. Many patents have been taken out both in Great Britain and abroad, some utilizing electrically recorded wave forms and a photoelectric cell, and others building up the tone quality from combinations of sine waves; for it is well known that any periodic wave can be analysed into a series of sine waves of which the frequencies are multiples of that of the original wave. Of the latter, the two most successful are that of the Compton Organ Co., in which the waves are generated by a variable capacity, and the Hammond organ.

In the Hammond organ the currents are produced electromagnetically by rotating an iron disk $D$ (Fig. 1), about the size of a half-crown, with a serrated edge, in front of the end of a permanent magnet $M$ on which is a coil of wire $B$. The change of flux through the coil, caused by the variation of the gap between the serrated edge of the disk and the end of the magnet, produces an alternating E.M.F. in the coil; the edge of the disk and the pole of the magnet are so shaped that this E.M.F. shall follow a sine curve as closely as possible. There are twelve of these disks, each with its own magnet and bobbin of wire, to each octave. The disks of one octave are all mounted on a single shaft and rotate as a whole. The shafts and disks of the successive octaves are all similar and are so geared together that the shaft of each octave shall run at twice the speed of the preceding one.

The number of serrations on the edges of the twelve disks of one of these octaves has to increase progressively so as to give as approximately as possible the notes of the equitempered scale. The number of turns on the bobbins is reduced in the successive octaves to compensate for the increased flux due to the increased speed of rotation, and by this as well as by adjusting the distance of the magnets from the edges of the disks, the intensities of the notes produced are equalized throughout the whole range of the circuits, of which there are ninety-one in all.

There are two keyboards, each with five.octaves. To combine the sine waves produced by the rotations of the disks in order to give the organ tones required, each note $K$ (Fig. 1) on the keyboard can be connected to nine harmonically related bobbins, and the intensity of each of these circuits can be varied at will. For example, middle $\mathrm{C}$ can be connected not only to the bobbin giving the note of middle $\mathrm{C}$, but also to the bobbins giving the fourth and the octave below $\left(G_{1}\right.$ and $\left.C_{1}\right)$ and to those giving the octave above, the twelfth, the second octave, seventeenth, nineteenth, and third octave $\left(\mathrm{C}^{\prime}\right.$,

Diagram of action of the Hammond organ : $A$, coll ; $B_{1}, B_{2}$, Bobbins ; Fig. 1. $C_{1}, C_{2}$, Capacities ; $D_{1}, \mathrm{D}_{2}$, DISKs ; $D B_{1}, D B_{2}$, DRAWBARS ; $E_{1}, E_{2}$, CON.

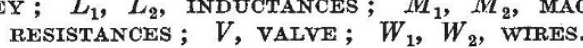

$\mathrm{G}^{\prime}, \mathrm{C}^{\prime \prime}, \mathrm{E}^{\prime \prime}, \mathrm{G}^{\prime \prime}, \mathrm{C}^{\prime \prime \prime}$.) These connexions are made by means of insulated contacts $E$ under the keys, one connected to each bobbin, which are brought down, when the key is depressed, on to nine wires $W$ stretching from side to side of the keyboard. The nine wires go to a group of nine draw-bars $D B$ situated over the keyboard, each of which can be set at nine positions numbered from zero to eight. All the ninety-one circuits have initially the same resistances, and the draw-bars insert a resistance $R$ which varies from infinity to nothing as they are drawn out, and thus each draw-bar adds a determined amount of the corresponding harmonic according to the position at which it is set.

To prevent the current from one bobbin feeding back through a key and the draw-bars to another bobbin and so giving false notes, there is a tuned circuit, consisting of a capacity and an inductance in parallel, between each bobbin and the keyboard. 
Thus the complete circuit starting from the bobbin passes first through the tuned circuit, then branches and goes to the nine keys connected to that bobbin. Then it goes through the wires stretched across the keyboard to the nine drawbars and through their resistances. These resistances are all joined together and the combined

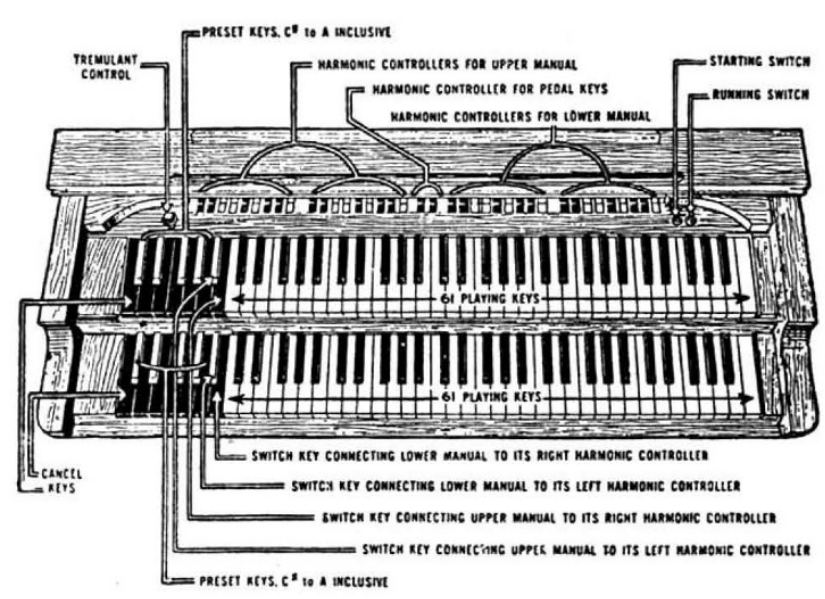

Fig. 2

current now goes to a coil $A$ which may be considered to be similar to the aerial coil of a wireless set, and from that back to the bobbins. The current is now amplified in the usual way and after a second amplification by another valve it passes to a variable resistance controlled by the swell pedal; this swell effects an enormous change in intensity, amounting to a variation of some fifty decibels as compared with about thirty at most of the ordinary organ. It, in fact, is equivalent to the drawing of extra stops in an ordinary organ in addition to the increase produced by the opening of the swell box. From the swell pedal resistance the current is taken by a cable to the power cabinet, where it is amplified by a

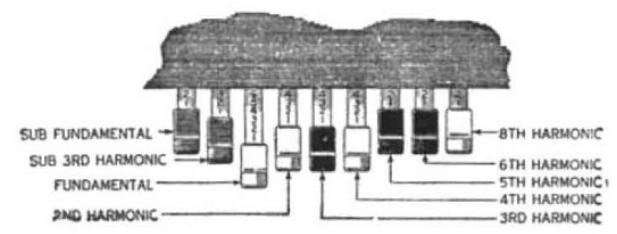

Fig. 3

further range of valves and feeds the loud-speakers, of which there may be two or four or more according to the size of the building for which the organ is required.

As already mentioned, there are two five-octave keyboards (see Fig. 2). Above the keyboards there are four groups of harmonic draw-bars, nine bars to each group; the two left groups are for the upper keyboard and the two on the right for the lower keyboard. One of these groups is shown in Fig. 3. Between these in the middle are two harmonic controllers for the pedals. To the left of the five octaves of playing keys is another group of keys looking like the playing keys except that their colours are reversed. These correspond to the piston buttons in an ordinary organ, and eighteen of them give various combinations of tones which imitate very closely the types of combinations that are obtained on an ordinary organ - the diapasons, the reeds, diapasons and reeds, full organ, certain solo stops, etc.--pressing down one of these keys brings that quality of tone into action, at the same time cancelling the last combination used. Two of the keys bring one or other of the two groups of drawbars over the keyboard into action; thus the player can change from one of these groups to the other instantly, or to any one of the nine pre-set combinations, merely by pressing the appropriate key. The great variation in power of 50 decibels given by the swell pedal makes each of these pre-set combinations equivalent to a large number of organ stops.

To indicate the use of the drawbars a few tone colours as given by the makers are set out below. The numbers indicate the extent to which the bars are drawn (third from left is the fundamental).
$007,755,220$ Open diapason.
$012,221,300$ Soft strings.
145,645,553 Full swell.
$015,656,342$ Diapason chorus with mixtures.
$006,470,520$ Clarinet.
$677,778,677$ Full organ.

It is obvious that an almost infinite number of combinations of these harmonics is possible, and thus an immense variety of musical tones can be imitated. The beauty of the quality of the diapason and flute tones is remarkable, and the full pedal notes come out excellently.

There can be no doubt that the loud-speaker electric organ in some form has come to stay. The small compass in which the equivalent of a large organ can be housed-the manual occupies no more room than a piano and the loud-speakers about that of a wireless set-the small cost, and the small running cost, together with the fact that it never requires tuning, are very real advantages which it possesses both for churches and for home use.

We are indebted to Messrs. Boosey \& Hawkes, Ltd., 295 Regent Street, London, W.1, for information on which the above article has been partly based. 